\title{
Epidemiology of Venous Thrombo-Embolic Disease in Yaounde: A Cross-Sectional Study in Sub-Saharan Africa
}

\author{
Sylvain Raoul Simeni Njonnou1, Chris Nadège Nganou-Ngnindjo1,2, Bâ Hamadou, ${ }^{1,2}$, \\ Bonaventure Jemea ${ }^{3,4}$, Ahmadou Musa Jingi ${ }^{1}$, Jerome Boombhi'1,5, Aimée Tiodoung Timnou ${ }^{1}$, \\ Marie-Josiane Ntsama Essomba1,2, Lydienne Alida Tonye1, Aicha Mefire Yap1, \\ Murielle Florence Helles Lema', Liliane Mfeukeu-Kuate ${ }^{1,2}$, Sylvie Ndongo Amougou, ${ }^{1,4}$, \\ Samuel Kingue1,5
}

\author{
${ }^{1}$ Department of Internal Medicine and Specialties, Faculty of Medicine and Biomedical Sciences, University of Yaounde I, \\ Yaoundé, Cameroon \\ ${ }^{2}$ Yaounde Central Hospital, Yaoundé, Cameroon \\ ${ }^{3}$ Department of Surgery and Specialties, FMSB, University of Yaounde I, Yaoundé, Cameroon \\ ${ }^{4}$ Yaounde University Teaching Hospital, Yaoundé, Cameroon \\ ${ }^{5}$ Yaounde General Hospital, Yaoundé, Cameroon \\ Email:raoulsims@yahoo.fr
}

How to cite this paper: Simeni Njonnou, S.R., Nganou-Ngnindjo, C.N., Hamadou, B., Jemea, B., Jingi, A.M., Boombhi, J., Tiodoung Timnou, A., Ntsama Essomba, M.-J., Tonye, L.A., Mefire Yap, A., Helles Lema, M.F., Mfeukeu-Kuate, L., Ndongo Amougou, S. and Kingue, S. (2019) Epidemiology of Venous Thrombo-Embolic Disease in Yaounde: A Cross-Sectional Study in Sub-Saharan Africa. World Journal of Cardiovascular Diseases, 9, 360-369.

https://doi.org/10.4236/wjcd.2019.95032

Received: April 26, 2019

Accepted: May 27, 2019

Published: May 30, 2019

Copyright (๑) 2019 by author(s) and Scientific Research Publishing Inc. This work is licensed under the Creative Commons Attribution International License (CC BY 4.0).

http://creativecommons.org/licenses/by/4.0/

\begin{abstract}
Background: Venous thromboembolic disease (VTE) is one of the main causes of cardiovascular death and a public health problem worldwide. It is one of the most complications in admitted patients, particularly in low-income settings. The epidemiological data on VTE are still lacking. Methods: We carried out a cross-sectional study in three hospitals in Yaoundé. We retrospectively reviewed records of patients admitted for VTE from January 2013 to December 2017. We collected data on socio-demography, clinical presentation, venous Doppler/pulmonary CT scan, and outcome. Results: We included 93 patients ( 43 males) with VTE. Their mean age was $53.3 \pm 16.6$ years. There were $46(49.5 \%)$ cases of Deep Vein Thrombosis (DVT), 36 (38.7\%) cases of Pulmonary Embolism (PE), and 11 (11.8\%) cases of PE associated with DVT. The main risk factors were obesity/overweight (58.1\%), immobility (43\%), HIV infection (22.6\%), prior admission (22.6\%), and long trip (19.4\%). The most frequent clinical presentation for PE was dyspnea (100\%), tachypnea $(87.2 \%)$, and chest pain $(70.2 \%)$. For DVT, limb pain (93\%), calf stiffness (86\%), limb volume $>3 \mathrm{~cm}(82.4 \%)$ were the most common presentation. There were $9(9.7 \%)$ in-hospital deaths. Mean hospital stay was $20.7 \pm 30.8$ days. Conclusion: VTE is underdiagnosed in our setting. Obesity and immobility were the main risk factors. Dyspnea and tachypnea
\end{abstract}


were the main clinical presentation for PE whereas limb pain and calf stiffness were the main symptoms for DVT. In-hospital mortality is still high.

\section{Keywords}

Venous Thromboembolic Disease, Epidemiology, Yaounde, Sub-Saharan Africa

\section{Introduction}

Venous Thrombo-Embolic disease (VTE) is recognized as a public health problem worldwide, affecting 78 to 137 million people yearly [1] [2]. It is the third most common cardiovascular disease according to the World Health Organization (WHO) [3]. Epidemiological data on VTE are heterogeneous, depending on diagnostic criteria and studied populations. The burden of VTE in low-income settings in sub-Saharan Africa (SSA) is not well known as data are scanty [4] [5] [6] [7] [8]. This burden is expected to be high due to the associated high rate of HIV infection [9]. In Africa, few studies have been carried out on VTE, generally on specific population [4] [6] [10] [11] [12]. Few data on VTE epidemiology exist in Cameroon, mainly from single centers, and usually, retrospective studies showing a predominance of Deep Venous Thrombosis (DVT) [8] [13] [14] [15]. Studies of VTE in Cameroon found prevalent DVT and male predominance (Kingue et al., Abah et al., and Kamdem et al.). The main risk factors were similar between studies-obesity, immobility, long trip in different proportions [8] [14]. Clinical presentations were the same: dyspnea and chest pain for pulmonary embolism (PE), and limb pain, calf stiffness, and swollen limb for DVT [8] [14] [15].

This cross-sectional study aimed at characterizing the epidemiological, clinical, imaging, and outcome specificities in patients with a diagnosis of VTE in three main hospitals in Yaounde, Sub-Saharan Africa (SSA).

\section{Methodology}

Study design and setting: This cross-sectional study was carried out in three teaching hospitals in Yaoundé: Yaoundé Central Hospital, Jamot Hospital, and Yaoundé University Teaching Hospital. These are tertiary hospitals in Yaoundé, the capital city of Cameroon (SSA). They have a catchment population of about 2 million inhabitants.

Study population: We retrospectively recruited patients admitted for VTE between January 2013 and December 2017 via their clinical records. We reviewed the clinical records of adult patients, aged $\geq 18$ years, of both sexes, admitted for VTE confirmed with Doppler ultrasound and/or pulmonary CT scan. Patients with incomplete files or files with missing key data were excluded.

Variables and Measurements: We collected data on socio-demography (age, sex, and profession), clinical presentation, venous Doppler/pulmonary CT scan, 
and outcome (survival versus death). Risk factors for VTE recorded were age $\geq$ 65 years, varicosity of the lower limbs, prolong rest/immobilization ( $>3$ weeks), high adiposity $\left(\mathrm{BMI}>25 \mathrm{Kg} / \mathrm{m}^{2}\right)$, pregnancy/early post-partum (<6 weeks), recent surgery, use of oral contraception in women, cancer, nephrotic syndrome, genetic thrombophilia, vascular trauma, antiphospholipid syndrome, and chronic inflammatory diseases. We also recorded the associated co-morbidities: Chronic Obstructive Pulmonary Disease (COPD), Asthma, and confirmed HIV (positive Western blot). Doppler ultrasound of the lower limbs was performed in case of suspicion of Deep Vein Thrombosis (DVT). Positive echographic criteria included direct signs (direct visualization of an endoluminal clot), and indirect signs (incompressibility with venous dilatation, upstream venous stasis, increased flow of collateral veins and alteration of venous flow). The localization and extension of thrombi were specified. Pulmonary CT scan was performed in case of suspected Pulmonary Embolism (PE) (clinical or echocardiographic). The diagnosis of $\mathrm{PE}$ included direct signs (direct visualization of endoluminal clot or defect), and indirect signs (oligemia, reduction in pulmonary arterial flow or vessel size). Intra-hospital evolution was recorded. It was of two types: favorable (improvement in clinical presentation and discharged home), or unfavorable (PE occurrence in case of DVT, intensive care referral or death). Complications related to treatment and hospitalization were also recorded (bed sores, infections, bleeding).

Ethical consideration: The institutional review board of the Faculty of Medicine and Biomedical Sciences of the University of Yaounde 1 approved this work. Administrative authorizations were obtained from the hospital administration. We carried out this work in accordance with the declarations of Helsinki. We report this work following the Standard for Reporting Observational Studies in Epidemiology (STROBE) checklist.

Sample size and Statistical analysis: A consecutive sample of all eligible cases were considered for this study. Data were analyzed using the software EPI INFO 3.5. We have presented discrete variables as counts and percentages, and continuous variables as mean (standard deviation). We used the Chi-square test, Student t-test, and ANOVA where appropriate. A p-value $<0.05$ was considered significant for the observed differences or associations.

\section{Results}

Participants: During the study period, 151 patient cases were identified as potential cases of VTE. We excluded 58 (missing files, incomplete files, and alternative diagnosis). Figure 1 presents the patient flow chart. Finally, 93 cases of VTE were included, of which $43(46.2 \%)$ were males. The mean age was $53.3 \pm$ 16.6 years (range from 17 to 89 years, $\mathrm{p}=0.40$ between sexes). People living with HIV (PLHIV) were younger $(\mathrm{p}=0.002)$. These patients were admitted in the internal medicine ward (78 cases) and in ICU (15 cases). Table 1 shows the general characteristics of this population.

Main data: There were $46(49.5 \%)$ cases of Deep vein thrombosis (DVT), 36 (38.7\%) cases of pulmonary embolism (PE), and $11(11.8 \%)$ cases of PE asso- 
ciated with DVT. Hypertension (26.9\%) and diabetes (9.7\%) were the most common comorbidities. The risk factors of VTE are presented in Table 2. Obesity/overweight (58.1\%), immobilization (43\%), prior admissions (22.6\%), HIV infection (22.6\%), long trip (19.4\%) were the most common risk factors.

Table 3 shows the clinical and para-clinical presentations, and treatment of the study population. The most frequent clinical presentation for PE was dyspnea $(100 \%)$, tachypnea $(87.2 \%)$, and chest pain $(70.2 \%)$. For DVT, limb pain (93\%), calf stiffness (86\%), limb volume $>3 \mathrm{~cm}(82.4 \%)$ were the most common presentations.

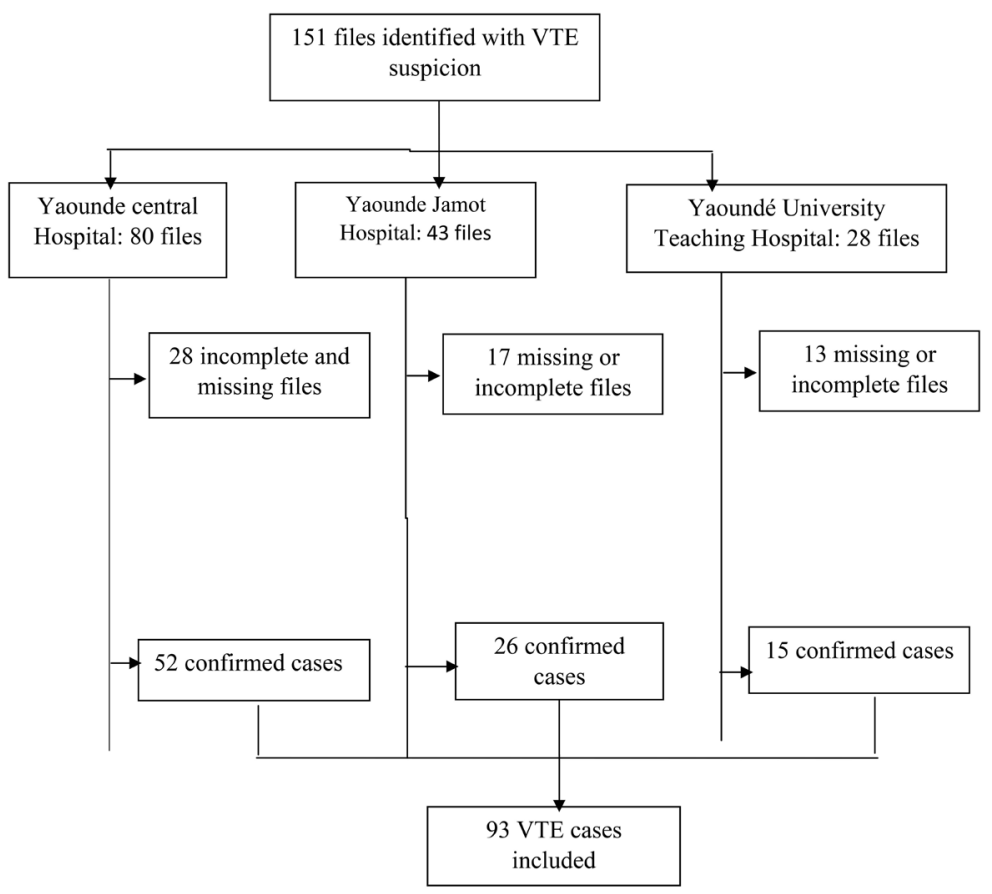

Figure 1. Patient flow chart.

Table 1. General characteristics of the study population.

\begin{tabular}{cc}
\hline Variables & Frequency (\%) \\
Gender & $43(46.2)$ \\
Male & $50(53.8)$ \\
Female & \\
Age group & $3(3.2)$ \\
$<20$ & $16(17.2)$ \\
$20-<40$ & $42(45.2)$ \\
$40-<60$ & $32(34.4)$ \\
$\geq 60$ & \\
Hospitalization ward & $78(83.9)$ \\
Medical & $15(16.1)$
\end{tabular}


Table 2. VTE risk factors in the study population.

\begin{tabular}{ccc}
\hline Risks factors & Frequency $(\%)$ & P value \\
\hline Old age & $26(28)$ \\
Immobility & $40(43)$ \\
Overweight/obesity & $54(58.1)$ \\
Varicose veins & $3(3.2)$ \\
Thrombosis past history & $4(4.3)$ \\
Puerperium & $4(4.3)$ \\
Surgery & $4(4.3)$ \\
Prior admission & $21(22.6)$ \\
Contraceptive use & $4(4.3)$ \\
Cancer & $4(4.3)$ \\
Long trip & $18(19.4)$ \\
Genetic thrombophilia & $2(2.2)$ \\
Chronic infectious and inflammatory diseases & $12(12.9)$ \\
HIV infection & $21(22.6)$ \\
Mean age of HIV patients & 43.6 \\
Mean age non HIV patient & 56.1 \\
\hline
\end{tabular}

Table 3. Clinical and para-clinical presentation and treatment of the study population.

\begin{tabular}{cc}
\hline Variables & Frequency (\%) \\
\hline DVT signs $(\mathrm{n}=57)$ & $53(93)$ \\
Limb pain & $49(86)$ \\
Calf stiffness & $47(82.4)$ \\
Limb volume $>3 \mathrm{~cm}$ & $41(71.9)$ \\
Homans sign & \\
PE signs (n $=47)$ & $47(100)$ \\
Dyspnea & $33(70.2)$ \\
Chest pain & $3(6.4)$ \\
Syncope & $17(36.2)$ \\
Cough & $9(19.1)$ \\
Hemoptysis & $41(87.2)$ \\
Tachypnea & $30(63.8)$ \\
Tachycardia & $13(48.1)$ \\
ECG (n = 27) & $1(3.7)$ \\
Tachycardia & $5(18.5)$ \\
Right bundle block & \\
S1Q3 pattern &
\end{tabular}




\section{Continued}

\begin{tabular}{|c|c|}
\hline Anterior $\mathrm{T}$ wave inversion & $7(25.9)$ \\
\hline ST modifications & $4(14.8)$ \\
\hline \multicolumn{2}{|c|}{ Localization on Doppler US $(\mathrm{n}=57)$} \\
\hline Soleal & $7(12.3)$ \\
\hline Tibial & $19(33.3)$ \\
\hline fibular & $12(21)$ \\
\hline Popliteal & $43(75.4)$ \\
\hline Femoral & $44(77.2)$ \\
\hline Iliac & $22(38.6)$ \\
\hline Inferior Vena Cava & $3(5.3)$ \\
\hline \multicolumn{2}{|l|}{ Localization on CT $\operatorname{scan}(\mathrm{n}=47)$} \\
\hline Troncular & $17(37)$ \\
\hline Lobar & $18(39.2)$ \\
\hline Segmental & $30(65.2)$ \\
\hline Sub Segmental & $14(24.6)$ \\
\hline \multicolumn{2}{|l|}{ VTE treatment $(n=93)$} \\
\hline Vitamin $\mathrm{K}$ antagonists & $56(60.2)$ \\
\hline Rivaroxaban & $30(32.2)$ \\
\hline НВРМ & $3(3.2)$ \\
\hline
\end{tabular}

All PE was confirmed by pulmonary angiography. Embolism was bilateral in most cases (56.5\%). Thrombus location was segmental in 30 (65.2\%) cases, lobar in $18(39.2 \%)$ cases, troncular in 17 (37\%) cases, and sub-segmental in $14(30.4 \%)$. In all PE, 27 ECG were performed with 16 being abnormal. The most frequent anomalies were sinus tachycardia in $13(48.1 \%)$ cases, $\mathrm{T}$ wave inversion in 7 (25.9\%) cases, S1Q3 pattern in $5(18.5 \%)$ cases, and ST modification in $4(14.8 \%)$ cases. DVT diagnosis were all confirmed by Doppler ultrasound. Main thrombi location were femoral in $44(77.2 \%)$ cases, popliteal in 43 (75.4\%), iliac in 22 (38.6\%) cases, and tibial in 19 (33.3\%) cases.

Most of the patients $(60.2 \%)$ were treated with anti-vitamin K (acenocoumarol and fluindione), 32.2\% with Rivaroxaban, $4.3 \%$ with Low-molecular-weight heparin (LMWH) and 3.2\% didn't receive any anticoagulant therapy. Thrombolysis was not used among study participants. Mean hospital duration was 20.7 days (ranged from 2 to 273 days).

Intra-hospital evolution was favorable in $74(79.6 \%)$ patients. Complications occurred in $19(20.4 \%)$ cases. Nosocomial infection (42.1\%) and hemorrhages (31.6\%) were the most frequent complications. There were 9 (9.7\%) cases of death. This was highest in patients with DVT (raising the suspicion of massive embolism). Hemorrhagic manifestations were associated with death $(\mathrm{p}=0.01)$. 


\section{Discussion}

This cross-sectional study aimed to describe the epidemiology, clinical presentations, treatment, and outcomes in patients having VTE in three teaching hospitals of Yaounde. Epidemiology of VTE is still poorly known in Africa. Few studies, especially on the prevalence and risk factors have been carried out [4] [5] [6] [7] [8] [10] [15] [16]. Our results show that Obesity/overweight, immobilization, prior admissions, HIV infection, long trip were the main risk factors for VTE. DVT was more common than PE.

The female predominance in this study is different from the reports of Kingue et al., Abah et al., and Kamdem et al. in Cameroon, but similar to that reported by Touré et al. and Kane et al. in Senegal [6] [8] [10] [13] [14]. The female predominance can be related to greater use of health facilities by women in Cameroon or selection bias. Patients' mean age is similar to that reported by Abah et al. and Kamdem et al., but higher than that reported by Awolesi et al., Kane et al., Kingue et al., and Touré et al., who reported a mean age of 40, 46.3, 46 and 42 years respectively [6] [8] [10] [11] [13] [14]. Goldstein has also described the younger age in South Africa [17]. Younger age in those publications may be related to the higher rate or HIV infection or sickle cell disease. In line with our previous report, HIV positive patients with VTE were younger [9].

The main risk factors were obesity/overweight (58.1\%), immobility (43\%), HIV infection (22.6\%), prior admission (22.6\%), and long trip (19.4\%). These results were partly similar to that reported by Kamdem et al. and Abah et al., who reported obesity, immobility, long trip as the most frequent risk factors [8] [14]. Surgical risk factors were the most frequently reported by Kingue et al. and Adeleye et al. in Cameroon and Nigeria respectively [13] [18]. There is a call for concern about HIV infection and its associated opportunistic affections, which is taking a huge place in many studies, and increasing with time [9] [10] [11]. This could be due to the fact that HIV, which is endemic in sub-Saharan Africa, especially in Eastern Africa, is associated with prothrombotic states, increased risk of tuberculosis which is also associated with VTE incidence (World report HIV, World report tuberculosis, Sullivan) [19] [20] [21]. In the setting of HIV infection, as in East or South Africa, Awolosei et al. and Goldstein et al. found tuberculosis as one of the main risk factors for DVT [11] [17]. Interestingly, despite the higher risk in a population with HIV or tuberculosis, HIV or tuberculosis are not included in the different thrombosis assessment scores (Wells and Geneva). These data differ from Asian findings where genetic factors are the main risk factors for VTE [22]. This racial disparity was described before by Zakai et al., may be due to lifestyle, diet (less obesity), and disease predominance [23].

The main clinical presentations of PE were dyspnea and chest pain, similar to that reported by Kamdem et al. [8]. Their high value may due to the milieu (patients were recruited in cardiology, pneumology, and ICU) where dyspnea and chest pain used to be common. The main DVT presentations were limb pain, 
calf stiffness, and swollen limb, similar in most other studies [5] [8] [11].

The mean hospital stay was quite long compared to that reported by Kamdem et al. [8]. This was due to the presence in our study of patients having tuberculosis and resistant tuberculosis leading to a long hospital stay. It was however similar to the hospital stay reported by Abah et al., Ondze-Kafata et al., and Aissa et al. [5] [14] [24]. Treatment with Rivaroxaban was more frequent among our patients. This is due to treatment simplification with this type of drug. Their prescription rate is expected to increase with time.

The mortality rate was $9.7 \%$ in this study, similar to that reported by Kamdem et al., and low compared to that reported by Abah et al., and Kingue et al. [13] [14]. This is due to the tertiary setting of our study, and also improvement of VTE management with time. Hemorrhagic complications were associated with death. It was only seen in patients with VKA. It raises the concern of a close follow-up of INR in those patients or the use of direct oral anticoagulants.

This study has some limitations due to the retrospective design, leading to a loss of many files of probable VTE patients or missing data. HIV serology was not routinely proposed to all patients with VTE.

\section{Conclusion}

VTE is underdiagnosed in our setting. Obesity and immobility were the main risk factors. Dyspnea and tachypnea were the main clinical presentation for PE, whereas limb pain and calf stiffness were the main symptoms of DVT. The main PE location was segmental and lobar. The most frequent thrombi location in DVT was femoral and popliteal. Mean hospital stay was 20.7 days. In-hospital mortality is still high.

\section{Authors' Contribution}

Conception and Design: SRSN, CNN, BH, BJ, SK. Data collection: CNN, SRSN, LAT, MY, MFHL, MJNE. Data analysis and Interpretation: SRSN, CNN, AM, BJ, BH, SK. Drafting of the manuscript: SRSN, CNN, AM, MJNE. All the authors read and approved the final draft for publication.

\section{Acknowledgements}

We thank the staff of Cardiology Unit in Yaounde Central Hospital, Pneumology Unit in Yaounde Jamot Hospital and Internal Medicine and ICU of Yaounde University Teaching Hospital.

\section{Conflicts of Interest}

The authors declare no conflicts of interest regarding the publication of this paper.

\section{References}

[1] Heit, J.A., Spencer, F.A. and White, R.H. (2016) The Epidemiology of Venous 
Thromboembolism. Journal of Thrombosis and Thrombolysis, 41, 3-14. https://doi.org/10.1007/s11239-015-1311-6

[2] ISTH Steering Committee for World Thrombosis Day (2014) Thrombosis: A Major Contributor to the Global Disease Burden. Journal of Thrombosis and Haemostasis, 12, 1580-1590. https://doi.org/10.1111/jth.12698

[3] Silverstein, M.D., Heit, J.A., Mohr, D.N., Petterson, T.M., O’Fallon, W.M. and Melton, L.J. (1998) Trends in the Incidence of Deep Vein Thrombosis and Pulmonary Embolism: A 25-Year Population-Based Study. Archives of internal medicine, 158, 585-593. https://doi.org/10.1001/archinte.158.6.585

[4] Kingue, S., Bakilo, L., Ze Minkande, J., Fifen, I., Gureja, Y.P., Razafimahandry, H.J.C., et al. (2014) Epidemiological African Day for Evaluation of Patients at Risk of Venous Thrombosis in Acute Hospital Care Settings: Cardiovascular Topic. Cardiovascular Journal of Africa, 25, 159-164. https://doi.org/10.5830/CVJA-2014-025

[5] Ondze-Kafata, L., Kouala, L.C., Traore-Kissima, A., Loulouamou, M., Bani, M., Amounya-Zobo, S., et al. (2012) La thrombose veineuse des membres inférieurs à Brazzaville: à propos de 44 cas. Cardiologie Tropicale, 135, 1-11.

[6] Touré, V.P., Abibatou, S., Pape Samba, B., Maboury, D., Moussa, S., et al. (2014) Risk Factors for Thrombosis in an African Population. Clinical Medicine Insights. Blood Disorders, 7, 1-6. https://doi.org/10.4137/CMBD.S13401

[7] Danwang, C., Temgoua, M.N., Agbor, V.N., Tankeu, A.T. and Noubiap, J.J. (2017) Epidemiology of Venous Thromboembolism in Africa: A Systematic Review. Journal of Thrombosis and Haemostasis, 15, 1770-1781.

https://doi.org/10.1111/jth.13769

[8] Kamdem, F., Ngahane, B.H.M., Hamadou, B., Mongyui, A., Doualla, M.S., Jingi, A.M., et al. (2018) Epidemiology, Clinical Presentations and In-Hospital Mortality of Venous Thromboembolism at the Douala General Hospital: A Cross-Sectional Study in Cameroon, Sub-Saharan Africa. World Journal of Cardiovascular Diseases, 8, 123-132. https://doi.org/10.4236/wjcd.2018.82012

[9] Simeni Njonnou, S.R., Nganou Ngnindjo, C.N., Bâ, H., Jemea, B., Jingi, A.M., Ntsama Essomba, M.J. and Kingue, S. (2018) Caractéristiques de la Maladie veineuse thromboembolique chez le sujet VIH positif à Yaoundé. La Revue de Médecine Interne, 39, A179. https://doi.org/10.1016/j.revmed.2018.03.136

[10] Kane, A., Pessinaba, S., Sanogo, A., Ndiaye, M.B., Mbaye, A., Bodian, M., et al. (2011) Maladie thrombo-embolique veineuse et infection à VIH : étude prospective à propos de 78 cas colligés à Dakar. Angéiologie, 63, 82-89.

[11] Awolesi, D., Naidoo, M. and Cassimijee, M.H. (2016) The Profile and Frequency of Known Risk Factors or Comorbidities for Deep Vein Thrombosis in an Urban District Hospital in KwaZulu-Natal. Southern African Journal of HIV Medicine, 17, 1-5. https://doi.org/10.4102/sajhivmed.v17i1.425

[12] Zabsonre, P., Nebie, L.V.A., Niakara, A., Samandoulougou, A., Toguyeni, J.Y. and Kabore, J.P. (2007) CO23-Maladie veineuse thrombo-embolique à Ouagadougou : place de l'infection à VIH. Annales de Dermatologie et de Vénéréologie, 134, 40. https://doi.org/10.1016/S0151-9638(07)89056-9

[13] Kingue, S., Tagny-Zukam, D., Binam, F., Nouedoui, C. and Muna, W.F.T. (2002) La Maladie veineuse thromboembolique au Cameroun (A propos de $18 \mathrm{cas}$ ). Médecine Tropicale, 62, 47-50.

[14] Abah, J.P., Menanga, A., Mbatchou, B.H., Minkande, J.Z., Akono, M.N. and Kingue, S. (2016) Pattern of Venous Thromboembolic Diseases in a Resources-Limited Set- 
ting in Cameroon. The Pan African Medical Journal, 23, 236-246.

https://doi.org/10.11604/pamj.2016.23.236.7034

[15] Owono Etoundi, P., Esiené, A., Bengono B., Roddy, Afane Ela, A. and Ze Minkande, J. (2015) La Maladie Thromboembolique Veineuse. Aspects Épidémiologiques et Facteurs de Risque dans un Hôpital Camerounais. Health Sciences and Diseases, 16, 1-4.

[16] Tonye, L.A. (2014) Longs voyages et maladie veineuse thromboembolique dans la Ville de Bamenda. Faculté de Médecine et des Sciences Biomédicales.

[17] Goldstein, L.N. and Wu, M.-T. (2018) A One Year Audit of Patients with Venous Thromboembolism Presenting to a Tertiary Hospital in Johannesburg, South Africa. African Journal of Emergency Medicine, 8, 12-15. https://doi.org/10.1016/j.afjem.2017.08.006

[18] Adeleye, A.O. and Ogun, G.O. (2016) Clinically Diagnosed Postoperative Venous Thromboembolism in a Neurosurgery Practice in Nigeria. World Neurosurgery, 89, 259-265. https://doi.org/10.1016/j.wneu.2016.01.069

[19] UNAIDS Global AIDS Update (2016) UNAIDS.

https://www.unaids.org/sites/default/files/media_asset/global-AIDS-update-2016_e $\underline{\text { n.pdf }}$

[20] Sharif-Kashani, B., Bikdeli, B., Moradi, A., Tabarsi, P., Chitsaz, E., Shemirani, S., Esmaili-Khansarif, M. and Masjedi, M.-R. (2010) Coexisting Venous Thromboembolism in Patients with Tuberculosis. Thrombosis Research, 125, 478-480. https://doi.org/10.1016/j.thromres.2010.01.014

[21] World Health Organization (2014) Global Tuberculosis Report. Geneva, Switzerland.

[22] Wang, K.-L., Yap, E.S., Goto, S., Zhang, S., Siu, C.-W. and Chiang, C.-E. (2018) The Diagnosis and Treatment of Venous Thromboembolism in Asian Patients. Thrombosis Journal, 16, 1-12. https://doi.org/10.1186/s12959-017-0155-Z

[23] Zakai, N.A., McClure, L.A., Judd, S.E., Safford, M.M., Folsom, A.R., Lutsey, P.L. and Cushman, M. (2014) Racial and Regional Differences in Venous Thromboembolism in the United States in 3 Cohorts. Circulation, 129, 1502-1509. https://doi.org/10.1161/CIRCULATIONAHA.113.006472

[24] Aissa, I., Rachdi, I., Ben Miled, K. and Ghedira, H. (2011) Incidence de la Maladie thromboembolique veineuse chez des Hommes admis dans un service de pneumologie pour affection respiratoire aiguë. Revue de Pneumologie Clinique, 67, 129-135. https://doi.org/10.1016/j.pneumo.2010.04.003 